\title{
Frictional Properties of Carbon Nanotube Films with a Load Range from Micronewton to Millinewton Using mm Size Balls
}

\author{
Hiroshi Kinoshita ${ }^{1)^{*}}$, Takashi Nakayama ${ }^{2)}$, Takahiro Sumitani ${ }^{2)}$, Kazuya Takeshita ${ }^{2)}$, \\ Masanori Seki ${ }^{1)}$, Masahiro Fujii ${ }^{1)}$ and Nobuo Ohmae ${ }^{2)}$ \\ 1)Department of Mechanical Engineering, Faculty of Engineering, Okayama University \\ 3-1-1 Tsushimanaka, Kita-Ku, Okayama 700-8530, Japan \\ 2)Department of Mechanical Engineering, Faculty of Engineering, Kobe University \\ 1-1 Rokkodai, Nada-Ku, Kobe 657-8501, Japan \\ *Corresponding author: kinoshita@mech.okayama-u.ac.jp
}

( Manuscript received 10 March 2012; accepted 4 August 2012; published 31 January 2013 )
(Presented at Technical Session in the International Tribology Conference Hiroshima 2011)

Tribological properties of a vertically aligned carbon nanotube (CNT) film with a thickness of approximately $700 \mu \mathrm{m}$ under millinewton and micronewton level loads were investigated using a $1 \mathrm{~mm}$ radius ball. At the beginning of frictional slidings, friction forces were high, and then decreased and seemed to reach relative steady state values, in both millinewton and micronewton load experiments. After the initial high frictions, top surface CNTs contacting with the counterpart ball on the CNT film were plastically deformed and bundled each other, which were observed to be flat by scanning electron microscopy (SEM). In the relative steady friction states, the friction forces basically increased with increasing loads. Under millinewton level loads, curves of the friction force as a function of the loads seemed to be parabolic. Surprisingly, although no adhesion forces were detected, frictions existed even at zero loads under micronewton loads.

Keywords: carbon nanotube films, microtribology, tribology, adhesion forces, counterpart ball size

\section{Introduction}

Carbon nanotubes (CNTs) consist of graphene sheets rolled to form seamless cylinders and have diameters from 1 to $100 \mathrm{~nm}$ and lengths of more than $2 \mathrm{~cm}$. CNTs have been studied for many researchers because of their excellent properties and potential applications. In the mechanical properties, although CNTs have high stiffness with the Young's modulus of more than $1 \mathrm{TPa}$ and high tensile strength values of more than $50 \mathrm{GPa}$, they can be elastically deformed under large lateral displacement [1-6].

Numerous CNTs synthesized on substrates appear to be thin films with carpet like-structure, which are called "CNT films". We have carried out tribological experiments of a CNT film, in which $6 \mu \mathrm{m}$ length multi-walled CNTs were vertically aligned, using a few tens $\mu \mathrm{m}$ radius tips, under $\mu \mathrm{N}$ level loads [7]. The tribological properties of the CNT film satisfy the Amontons-Coulomb's friction law such as conventional thin films; however, the friction coefficients of the CNT film were extremely high (friction coefficient $\mu$ was over 1) and no wear was observed. One of the possible models to explain the high friction is proposed, in which the high frictions arises from high repulsive forces due to the bending of vertically aligned CNTs. There are some frictional experiments on CNT films carried out by other researchers. Friction coefficients on vertically aligned mainly double-walled CNT films with a thickness of below $300 \mathrm{~nm}$ under normal loads from about 10 to $100 \mathrm{nN}$ are measured using an atomic force microscope (AFM) with a about $20 \mathrm{~nm}$ radius tip [8]. In the $\mathrm{nN}$ load experiments using the $\mathrm{nm}$ radius tip, the nonlinearity relationships between the friction force and the normal load are observed, which might come from the buckling of the CNTs, and the high friction coefficients of 0.6-1.0 are obtained. However the reason of the high friction coefficient was not indicated. The friction experiments on the 40-80 $\mu \mathrm{m}$ thick vertically aligned multi-walled CNT film and 2-10 $\mu \mathrm{m}$ thick horizontally aligned multi-walled CNT film are carried out using a $0.778 \mathrm{~cm}$ radius borosilicate crown glass with the loads of $0.5-10 \mathrm{mN}$ [9]. The friction coefficient is 0.795 for the vertically aligned CNT film and 0.090 for the horizontally aligned CNT film. The $\mathrm{mN}$ load experiments with the $\mathrm{cm}$ radius tip demonstrate that the vertically aligned configuration is necessary for the high friction of CNT films. It is reported that in the friction 
condition with normal forces of 0.11-0.52 $\mathrm{N}$ using 2.4 $\mathrm{mm}$ radius SUS440C, the friction coefficients of the multi-walled CNT coatings synthesized vertically on SiC substrates are below 0.1 [10]. After the friction tests the CNT coatings are remained on the substrate, however fallen down and deformed. Namely, under the high loads, the counterpart tip slid sidewalls of CNTs, which would lead to the relative low friction coefficients. The tribological properties of $2.5 \mathrm{~nm}$ length single-walled CNT bundles are studied using classical molecular dynamic simulations [11]. In the simulations, the diamond surface acted as the counterpart surface slides the top end of the vertically aligned caped CNT bundle and the side walls of the horizontally aligned CNT bundle. The simulation predicts that the vertically aligned CNT bundle has much high friction than the horizontally aligned CNT bundle at high compression of over $10 \mathrm{GPa}$, in which the vertically aligned CNT bundle is plastically deformed with buckling, whereas the horizontally aligned CNT bundle were plastically deformed to a flat plate. We also carried out the micronewton load experiments using vertically aligned 30-70 $\mu \mathrm{m}$ multi-walled CNT films and approximately $20 \mu \mathrm{m}$ radius tip under a load of $\sim 5 \mu \mathrm{N}$ [12]. In the experiment, the high friction coefficients were also observed; however, in contrast to our experiment using the $6 \mu \mathrm{m}$ length CNT film above mentions, the wear tracks on the topmost surface of the CNT film surfaces were observed. In detailed, the top ends of the CNTs were plastically deformed, whereas the other part of the CNTs except for the deformation parts was still aligned vertically. This means that although the counterpart tip would slide the side walls on the deformed top end of the CNTs, the friction forces were high.

It is briefly summarized that the frictional experiments of CNT films using the $\mathrm{nN}$ loads and $\mathrm{nm}$ radius tip [8], the $\mu \mathrm{N}$ loads and $\mu \mathrm{m}$ radius tips [7,12], the $\mathrm{mN}$ loads and $\mathrm{cm}$ radius tip [9], and the $\mathrm{N}$ loads and $\mathrm{mm}$ radius tip [10], and the simulation [11] have been conducted. The findings in these experiments and simulation indicate that the properties of CNT films are much different in the different frictional conditions and the vertically aligned configurations of CNTs are necessary for the high frictions of CNT films (the slidings on top ends of vertically aligned CNTs is not necessary). However, the detailed mechanisms of high frictions of CNT films still remain unresolved. Since pressures on contact points on CNT film surfaces are related on bending, buckling, and deformation of CNTs, they are thought to be important. In order to investigate the effect of the pressure, the tribological properties of a CNT film were measured at load ranges of $\mu \mathrm{N}$ and $\mathrm{mN}$ using a $1 \mathrm{~mm}$ radius ball, which frictional condition have not yet conducted.

\section{Experiments}

In this study, the formation of CNT film on silicon oxide film substrate was carried out using thermal chemical vapor deposition (CVD). In the process, at first Fe layers with a thickness of $4 \mathrm{~nm}$ were deposited on the substrates, and then Fe nanoparticles were formed using an annealing treatment with condition of $750^{\circ} \mathrm{C}$ in $30 \mathrm{~Pa}$ $\mathrm{H}_{2}$ flow, for $40 \mathrm{~min}$. The thermal CVD process for the formation of the multi-walled CNT film was carried out with gas mixture of $\mathrm{C}_{2} \mathrm{H}_{2}$ and $\mathrm{H}_{2}$, at substrate temperature of $750^{\circ} \mathrm{C}$, for $20 \mathrm{~min}$, and at pressure of 300 $\mathrm{Pa}$. The density of the CNT film was approximately 300 $\mathrm{CNTs} / \mu \mathrm{m}^{2}$. The thickness of the CNT film was approximately $700 \mu \mathrm{m}$. Basically CNTs in the CNT film were vertically aligned, but at higher magnification, tangle structures were clearly observed by scanning electron spectroscopy (SEM) as shown below.

In experiments under millinewton level loads, a home-built millitribometer with two sets of parallel leaf springs for millinewton level lateral and normal force measurements was used. Frictional slidings were carried out in a reciprocating sliding configuration with a sliding speed of approximately $1 \mathrm{~mm} / \mathrm{s}$ and a sliding width of $1 \mathrm{~mm}$. A SUJ2 bearing ball with a radius of 1 $\mathrm{mm}$ was used as a counterpart ball in the experiments.

In the micronewton load experiments, a home-build microtribometer with a cantilever, with which $1 \mathrm{~mm}$ radius SUJ2 bearing ball was attached, and an optical lever system for detecting micronewton range lateral and normal forces was used; this was similar to the setup for conventional atomic force microscopy (AFM). The frictional experiments were conducted in a reciprocating sliding configuration with a sliding speed of approximately $90 \mu \mathrm{m} / \mathrm{s}$ and a sliding width of $90 \mu \mathrm{m}$. In the micronewton load experiments the same size SUJ2 ball as the millinewton load experiments was also used.

Normal loads corresponded directly to normal forces detected by the home-build millitribometer and microtribometer. In both the tribometers, normal loads applied were maintained at reference loads by a feedback controller, which adjusted z-pzt stretches. Since both the home-build millitribometer and microtribometer used the reciprocating motion configurations for frictions, friction forces were estimated from a half of a difference between an average value of forward lateral forces and an average value of backward lateral forces [13].

The counterpart balls were cleaned by an ultrasonic cleaner in acetone for $5 \mathrm{~min}$, ethanol for $5 \mathrm{~min}$, and water for $5 \mathrm{~min}$, and finally dried in air. Frictional experiments were carried out in air at a relative humidity of approximately $50-60 \%$, and a temperature of $24-27^{\circ} \mathrm{C}$.

\section{Results}

\subsection{Under millinewton level loads}

Figure 1 shows friction force of the CNT film as a function of sliding cycles with a load of $1 \mathrm{mN}$. At the beginning, friction force was over $1.3 \mathrm{mN}$ and decreased rapidly. And then although the friction force 


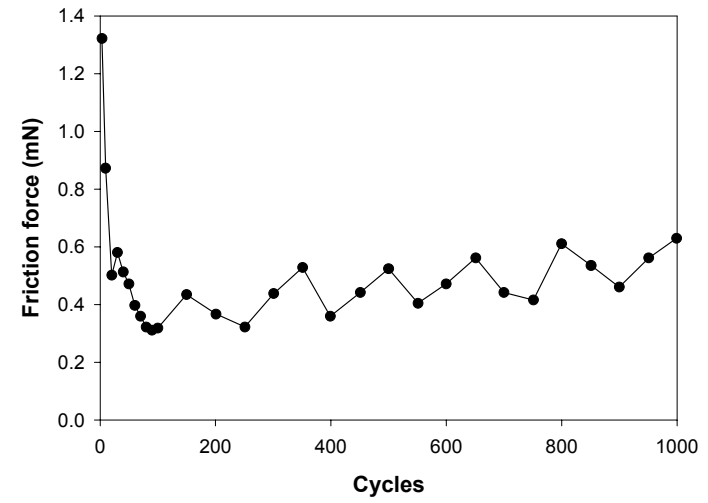

Fig. 1 Friction force as a function of friction cycles with the load of $1 \mathrm{mN}$

looks like slightly increasing, the friction force seemed to be relatively stable. SEM images of the CNT film surface before and after the initial high frictions using a $25 \mathrm{keV}$ electron beam are shown in Fig. 2. As mention above, CNTs in the CNT film were basically vertically aligned at low magnification. However the SEM image before the frictions clearly shows that the CNT film surface had tangle structure. This indicates that the counterpart ball slid the side walls of the laying CNTs. The SEM image after the initial high frictions shows that CNTs contacting with the counterpart ball were plastically deformed and bundled each other, which seems to be flat. The areas which did not contact with the counterpart ball were not deformed and maintained CNT fibrous structure. Only the topmost surface was deformed, and the inside of the CNT film seems not to be damaged. The flat planes were thought to consist of carbon atoms, not iron deriving from the counterpart ball. The SEM image after the initial high friction shows CNTs behind the flat planes, i.e., the flat planes were transparent. Electron energy of $25 \mathrm{keV}$ is enough high to go through thin planes consisted with light atoms, not heavy atoms. This would indicate that the flat planes consisted of carbon atoms. As mentioned above, the simulation predicts that the horizontally aligned CNT bundle is plastically deformed and the formation of plane layers of carbon atoms is occurred at the high compression [11]. In this study, the similar phenomenon would be occurred. The counterpart ball would compress the laying CNTs, and the compressed CNTs would be plastically deformed to the flat planes. The initial high friction was observed ant it arose from the plastic deformation. It is possible that after the initial high friction slight plastic deformation was occurred continuously and the number of contacting CNTs on the counterpart ball was increased, which caused the slightly increasing friction force.

Fig. 3(a) demonstrates the raw lateral and normal forces data under millinewton level loads. After 1000 cycle friction with the load of $1.05 \mathrm{mN}$ (in order to reach the relative steady friction state), the data was

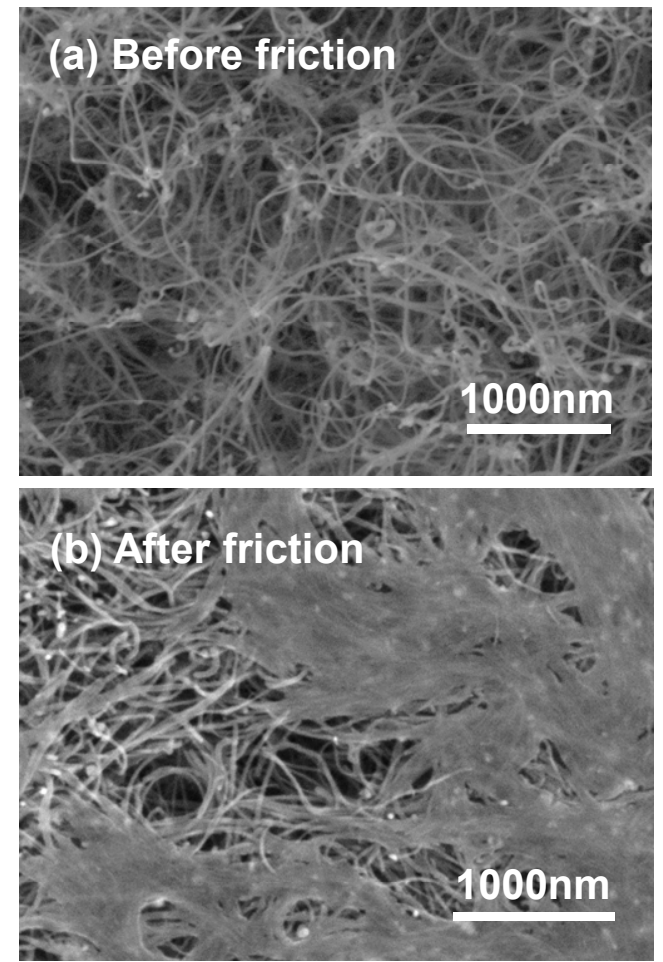

Fig. 2 SEM images of CNT film surfaces before and after the initial high friction under millinewton level loads

obtained as follows; with keeping frictional scan the reference load was set at $1.05 \mathrm{mN}$ and decreased gradually, decreasing by $0.08 \mathrm{mN}$, until zero value. Forward and backward lateral forces were observed due the reciprocating sliding configuration. The friction force decreased with decreasing the load. The detected normal load never became less than zero value even when the reference load was less than zero value (not shown), indicating that adhesion force was not detected (If adhesion force existed, a negative normal force would be detected). Fig. 3(b) shows the friction force as a function of the load. The curve seems to be parabolic. In loads of more than $0.2 \mathrm{mN}$, the friction seems to increase commensurately with the load. The friction coefficient estimated from the proportional constant of the curve shown in Fig. 3(b) was approximately 0.6. When friction coefficients were measured on different places of the CNT film surface, the friction coefficients ranged from 0.4 to 1.1 . It is possible that the wide variation of the friction coefficient might come from the different fibrous structures of the CNT film surface at the different measured places.

\subsection{Under micronewton level loads}

Initial high frictions with a friction coefficient of more than 1 and then relative stable frictions with lower friction coefficients were also observed in the micronewton load experiments. A SEM image of the CNT film surface obtained after the initial high frictions 


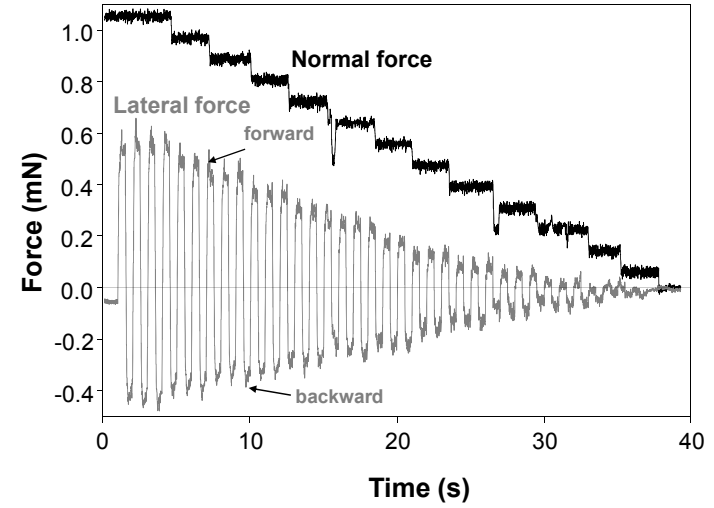

(a) Raw lateral and normal forces

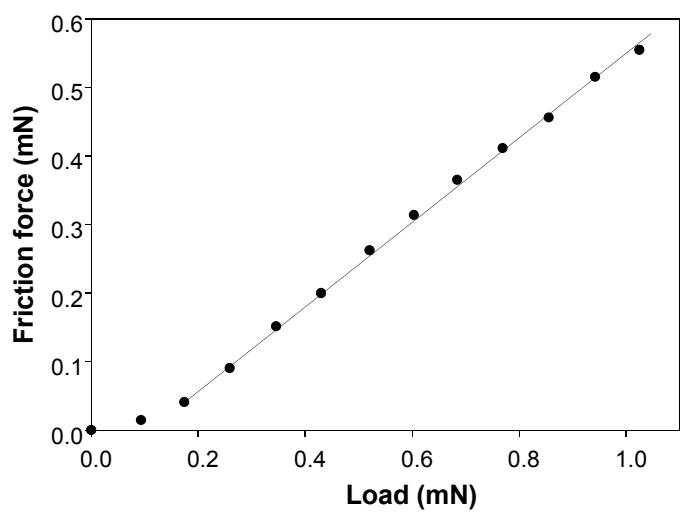

(b) Friction force vs. Load

Fig. 3 Friction and load data obtained under millinewton level loads, in the relative steady friction states. (a) Typical raw lateral and normal forces. The black line shows the normal force and the gray line shows the lateral force. (b) The friction force as a function of the load

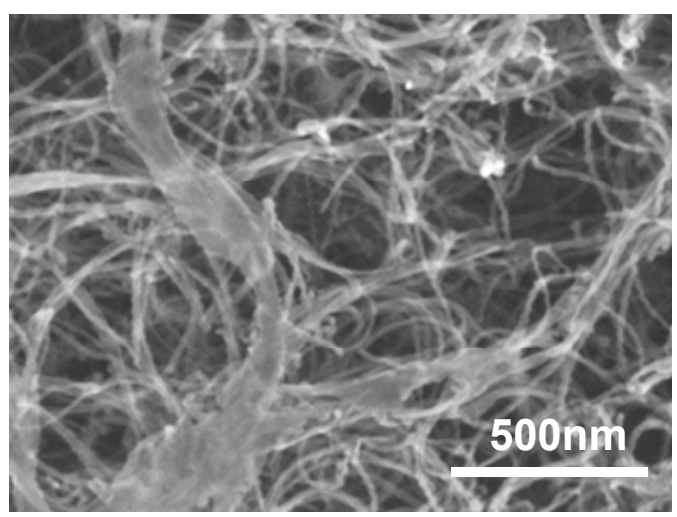

Fig. 4 SEM image of the CNT film surface with the load of $1 \mu \mathrm{N}$, in the relative steady friction states

is shown in Fig. 4. Plastic deformations and flat areas are also appeared on the CNT film surface. The initial high frictions under micronewton level loads were caused in the same manner as the millinewton load

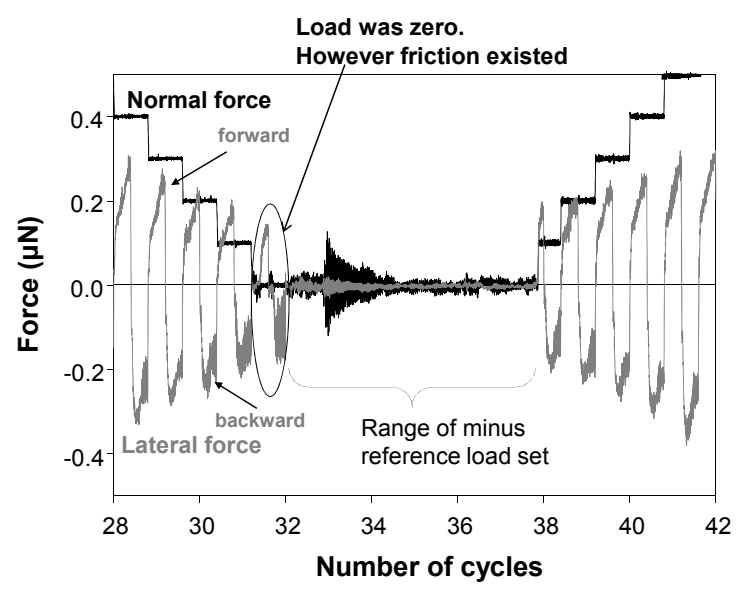

(a) Raw lateral and normal forces

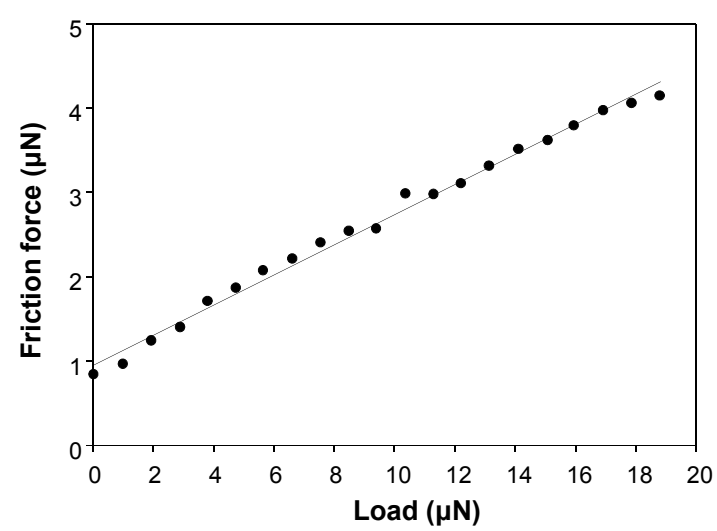

(b) Friction force vs. Load

Fig. 5 Friction and load data as the loads were micronewton range, in the relative steady friction states. (a) Typical raw lateral and normal forces. The black line shows the normal force and the gray line shows the lateral force. (b) The friction force as a function of the load

experiments. The flat area in Fig. 4 was smaller than that in Fig. 2(b), which would result from the lighter load.

Figure 5(a) shows typical raw lateral and normal forces data measured after 1000 cycle friction at the load of $2 \mu \mathrm{N}$. The procedure of the data acquisition was as follows; at first with keeping frictional scan the reference load was set at $2 \mu \mathrm{N}$ and decreased gradually, decreasing by $0.1 \mu \mathrm{N}$ /one scan (one set of the forward and backward scans), until minus value (actually -0.3 $\mu \mathrm{N}$ ), and then the reference load increased gradually, increasing by $0.1 \mu \mathrm{N}$, until the reference load of $2 \mu \mathrm{N}$. The graph shown in Fig. 5(a) is a close-up view around the zero normal force. The reciprocating sliding configuration in the microtribometer provided forward and backward friction forces. The friction force decreased with decreasing the load. The detected normal load never became less than zero value, indicating that adhesion force was not detected. When the load reached 
the zero value, the friction was clearly observed and then after one scan it was disappeared. When the load increased from the zero value, the friction force was appeared at a load of $0.1 \mu \mathrm{N}$. As mentioned above, the applied load was maintained at the reference load by the feedback controller. When the reference load was set at minus value (as indicated in Fig. 5(a)), the counterpart ball removed with the CNT film surface and the measured load was still zero. The relative large and small normal force oscillations (derived from vibration noises) were observed at the zero normal forces in Fig. 5(a). In order to confirm whether the ball contacted with the surface or not, the normal force oscillation was available. Since the counterpart ball was attached with the cantilever, the cantilever oscillated freely as the ball didn't contact with anything. Thus, the normal force oscillation was relatively large. While the ball contacted with the CNT film surface, the normal force oscillation was relatively small due to that the ball was sustained by the surface. When the normal force oscillation was relatively small, the friction force existed. When the normal force oscillation was relatively large, there was no friction force. This means that even though the zero loads were measured, the counterpart ball contacted with the CNT film surface and the friction force existed.

Fig. 5(b) shows the friction force as a function of the load in the relative stable friction state. The friction increased with proportional to the load, and the straight line goes to the positive y-axis, not to the origin. This is usually explained by adhesions between counterpart balls and sample surfaces. However any adhesion forces were not detected in the experiments. We previously carried out the frictional experiments of vertically aligned CNT films using micrometer radius tips, in which friction force was proportional to load and was not existed at zero loads. Therefore the much larger counterpart ball used in this study than the previous experiments (relatively lower compression) probably resulted in the friction at the zero loads. The friction coefficient calculated from the proportional relationship shown in Fig. 5(b) was 0.2. However, the friction coefficients obtained on the different areas of the CNT film surface were ranged from 0.2 to 2.0 . These wide values also might arise from the difference surface structures of CNT at the different measured places.

\section{Discussion}

It has been reported that CNTs have high stiffness, and are extraordinarily flexible under large strains [1-6]. However, in this study, the SEM images show that the CNTs contacting with the counterpart ball were plastically deformed and bundled each other, and appeared to be flat after the frictional slidings in both the millinewton and micronewton load experiments. Figure 6 illustrates the model of the frictional configuration in this study as following; the laying CNTs shown in Fig. 2(a) were bundled each other, and

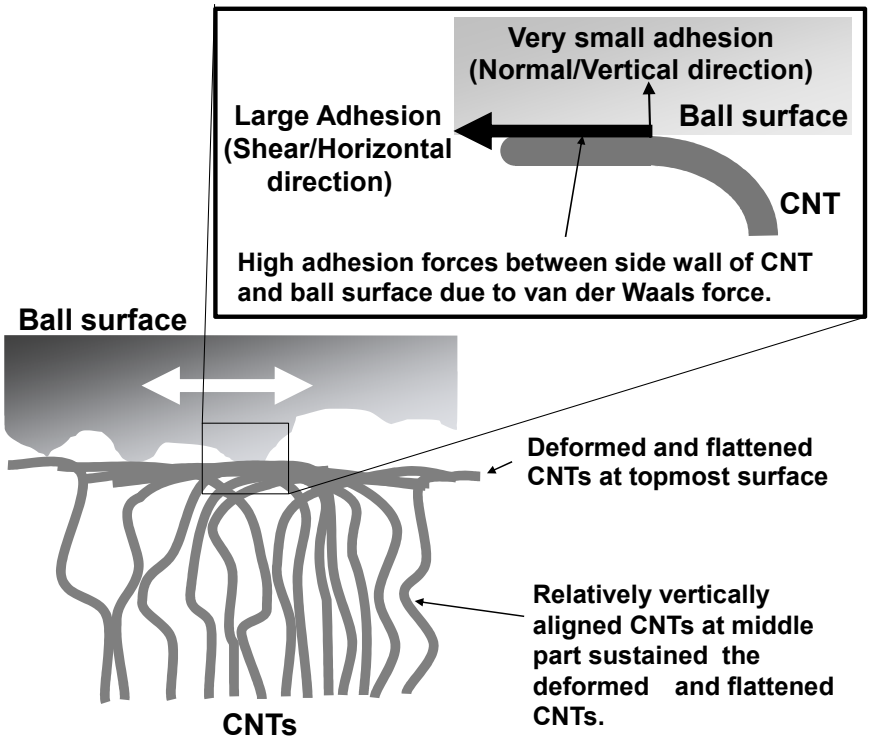

Fig. 6 Model of the frictional configuration in this study. Inlet shows the detailed configuration that the counterpart ball contacted with the sidewall of the deformed and flattened CNTs

flattened. The top surfaces of the flattened CNTs were sustained by a middle part of the vertically aligned CNTs, which were low hardness due to the CNTs fibrous structure. The counterpart ball surfaces contacted with the side walls of the flattened CNTs, which is shown in the inlet of Fig. 6.

Although the friction coefficients estimated by the linear relationships between the loads and the friction forces at different places were widely distributed in both millinewton and micronewton experiments, the friction coefficients obtained in this study were relatively high. As discussed above, counterpart surfaces slide on sidewall of the horizontally aligned CNTs, which leads to low frictions. In this study, the counterpart ball slid on the side walls of the deformed and flattened CNTs. The differences of the friction coefficients on the horizontally aligned CNT film (relative low) and this study (relative high) would arise from that the horizontally aligned CNTs are on the rigid substrate and the flattened CNTs in this study were sustained by the middle CNTs with low hardness. Therefore it is probably that in this study the middle parts of the CNTs, which were not deformed, affected to the high frictions, namely, the repulsive forces due to the bending of the middle CNTs caused the high frictions.

The curves of friction forces as a function of the loads seemed to be parabolic under the $\mathrm{mN}$ load experiments. The reason of the parabolic curve is probably as following; the ball applied by the relative high loads dented the CNT film, which was very soft due to the fibrous form of CNTs. The denting became a resistance as the counterpart ball moved, and the denting depth increased in increasing the load. 
Consequently, the friction force would doubly increase in increasing the load.

It is surprised that the friction existed at no loads although any adhesion forces were not detected. To our best knowledge, this phenomenon is never seen. Qu et al. reported that the strong anisotropic adhesion forces between a sidewall of CNTs and a substrate in shear and normal directions due to van der Waals force [14]. They explain that when CNTs which sidewalls contact with the substrate are vertically lifted off from the substrate, CNTs are easy to be removed from substrate due to the point-by-point peel-off detachment; on the contrary, when the contacting CNTs are horizontally dragged against the substrate, the adhesion forces are strong due to no peel-off detachment, i.e., it is hard to remove CNTs in horizontal direction (see inlet in Fig. 6). In this study under micronewton level loads, number of the CNTs which sidewalls contacted with the ball was much larger than the previous frictional experiment using the micrometer radius tips. Thus, under micronewton level loads, the total adhesion force along vertical direction was very small. In contrast, the total adhesion forces at horizontal direction became large, which provided the friction at no loads. The strong adhesion force of CNTs along horizontal direction and large number of the contacting CNTs under micronewton loads using the 1 $\mathrm{mm}$ radius ball would result in the friction at no loads without any detected adhesion forces.

\section{Summary}

Using the $1 \mathrm{~mm}$ radius balls, tribological properties of the vertically aligned CNT films at the load range from micronewton to millinewton were investigated. Under both micronewton and millinewton level loads, following the initial high frictions which would arise from the plastic deformations of the CNTs, the relative steady friction states with the relative high frictions were observed. The SEM images show that after the initial high friction top surface laying CNTs contacting with the counterpart ball were plastically deformed and bundled to flat planes, and the middle part of CNTs were not damaged. These experimental results are same results as the previous our $\mu \mathrm{m}$ radius tip and $\mu \mathrm{N}$ loads experiments using vertically aligned $30-70 \mu \mathrm{m}$ lengths CNT films, whereas deformation of CNT film surface was not observed using vertically aligned $6 \mu \mathrm{m}$ lengths CNT films. In contrast to the previous our $\mu \mathrm{m}$ radius tip and $\mu \mathrm{N}$ load experiments using both the $6 \mu \mathrm{m}$ and 30-70 $\mu \mathrm{m}$ lengths CNT films, the frictional behaviors under micronewton and millinewton level loads using $1 \mathrm{~mm}$ ball did not satisfy with the Amontons-Coulomb's friction law. Under millinewton loads, at the relative steady friction states, the friction force curve as a function of the load appeared to be parabolic. This probably resulted from the ball denting applied by the relative high loads and the low hardness of CNT films. The friction was observed at the zero loads without any detected adhesion forces under micronewton level loads. This would arise from the strong adhesion force of the deformed and flattened CNTs along horizontal direction.

\section{Acknowledgment}

Part of this study was supported by Grant-in-Aid for Scientific Research for Young Scientists (A) (20686013). The authors are grateful to M. Kageyama, T. Ohshima, and K. Toda for technical assistances and A. A. Alias for a critical reading of the manuscript.

\section{References}

[1] Wong, E. W., Sheehan, P. E. and Lieber, C. M., "Nanobeam Mechanics: Elasticity, Strength, and Toughness of Nanorods and Nanotubes," Science, 277, 1997, 1971-1975.

[2] Yu, M.-F., Lourie, O., Dyer, M. J., Moloni, K., Kelly, T. F. and Ruoff, R. S., "Strength and Breaking Mechanism of Multiwalled Carbon Nanotubes under Tensile Load," Science, 287, 2000, 637-640.

[3] Salvetat-Delmotte, J. P. and Rubio, A., "Mechanical Properties of Carbon Nanotubes: a Fiber Digest for Beginners," Carbon, 40, 2002, 1729-1734.

[4] Qi, H. J., Teo, K. B. K., Lau, K. K. S., Boyce, M. C., Milne, W. I., Robertson, J. and Gleason, K. K., "Determination of Mechanical Properties of Carbon Nanotubes and Vertically Aligned Carbon Nanotube Forests Using Nanoindentation,’ Journal of the Mechanics and Physics of Solids, 51, 11-12, 2003, 2213-2237.

[5] Coleman, J. N., Khan, U., Blau, W. J. and Gun'ko, Y. K., "Small But Strong: A Review of the Mechanical Properties of Carbon Nanotube-Polymer Composites," Carbon, 44, 9, 2006, 1624-1652.

[6] Falvo, M. R., Clary, G. J., Taylor, R. M., Chi, V., Brooks Jr, F. P., Washburn, S. and Superfine, R., "Bending and Buckling of Carbon Nanotubes under Large Strain.," Nature, 389, 1997, 582-584.

[7] Kinoshita, H., Kume, I., Tagawa, M. and Ohmae, N., "High Friction of a Vertically Aligned Carbon-Nanotube Film in Microtribology," Applied Physics Letters, 85, 14, 2004, 2780-2781.

[8] Miyake, K., Kusunoki, M., Usami, H., Umehara, N. and Sasaki, S., "Tribological Properties of Densely Packed Vertically Aligned Carbon Nanotube Film on SiC Formed by Surface Decomposition," Nano Letters, 7, 11, 2007, 3285-3289.

[9] Dickrell, P. L., Sinnott, S. B., Hahn, D. W., Raravikar, N. R., Schadler, L. S., Ajayan, P. M. and Sawyer, W. G., "Frictional Anisotropy of Oriented Carbon Nanotube Surfaces," Tribology Letters, 18, 1, 2005, 59-62.

[10] Hirata, A. and Yoshioka, N., "Sliding Friction Properties of Carbon Nanotube Coatings Deposited 
by Microwave Plasma Chemical Vapor Deposition," Tribology International, 37, 11-12, 2004, 893-898.

[11] Ni, B. and Sinnott, S. B., "Tribological Properties of Carbon Nanotube Bundles Predicted from Stomistic Simulations," Surface Science, 487, 1-3, 2001, 87-96.

[12] Kinoshita, H., Matsumoto, N. and Ohmae, N., "High Frictions of Vertically Aligned and Spaghetti-Like Carbon Nanotube Films in Microtribology," International Tribology
Conference (Austrib 2006) CD-ROM, 2006, Paper No. 303.

[13] Cain, R. G., Reitsma, M. G., Biggs, S. and Page, N. W., "Quantitative Comparison of Three Calibration Techniques for the Lateral Force Microscope," Review of Scientific Instruments, 72, 8, 2001, 3304-3312.

[14] Qu, L., Dai, L., Stone, M., Xia, Z. and Wang, Z. L., "Carbon Nanotube Arrays with Strong Shear Binding-On and Easy Normal Lifting-Off.," Science, 322, 2008, 238-242. 